\title{
A Large Intestine Disorder: Hirschsprung's Disease
}

Seyyed Mohsen Hosseininejad ${ }^{1,2}$, Fateme Sadat Hosseininejad ${ }^{3}$ and Seyyed Ahmad Hosseini ${ }^{2 *}$

${ }^{1}$ Golestan University of Medical Sciences, Gorgan, Iran

${ }^{2}$ Pediatric Neurology Department, Neonatal and Children Health Research Center, Golestan University of Medical Sciences, Gorgan, Iran

${ }^{3}$ School of Public Health, Shahroud University of Medical Sciences, Shahroud, Iran

\section{Retraction Note:}

The article entitled "A Large Intestine Disorder: Hirschsprung's Disease" has been accepted for publication in the International Journal of Clinical and Medical Images considering the statements provided in the article as personal opinion of the author which was found not having any conflict or biasness towards anything. As the article was a perspective one, information provided by the author was considered as an opinion to be expressed through publication.

Publisher took decision to make the article online solely based on the author's decision. However, it is found that the author have some personal concerns and issues, therefore being retracted from the journal.

Author Comments: Kindly retract the manuscript online because I am developing the manuscript and extending the text. I will resend it after I finished it.

*Corresponding author: Seyyed Ahmad Hosseini, Pediatric Neurology Department, Neonatal and Children Health Research Center, Golestan University of Medical Sciences, Gorgan, Iran,Tel: +981732253500;E-mail: parnianah@yahoo.com 